\title{
Competition in Private Commons: Price War or Market Sharing?
}

\author{
Emir Kavurmacioglu, Murat Alanyali and David Starobinski \\ Division of Systems Engineering, Department of Electrical Engineering \\ Boston University \\ \{emir, alanyali, staro\}@bu.edu
}

\begin{abstract}
This paper characterizes the outcomes of secondary spectrum markets when multiple providers compete for secondary demand. We study a competition model in which each provider aims to enhance its revenue by opportunistically serving a price dependent secondary demand, while also serving dedicated primary demand. We consider two methodologies for sharing spectrum between primary and secondary demand: In coordinated access, spectrum providers have the option to decline a secondary access request if that helps enhance their revenue. We explicitly characterize a break-even price such that profitability of secondary access provision is guaranteed if secondary access is priced above the break-even price, regardless of the volume of secondary demand. Consequently, we establish that competition among providers that employ optimal coordinated access leads to a price war, as a result of which the provider with the lowest break-even price captures the entire market. This result holds for arbitrary secondary demand functions. In uncoordinated access, primary and secondary users share spectrum on equal basis, akin to ISM bands. Under this policy, we characterize a market sharing price which determines a provider's willingness to share the market. We show an instance where the market sharing price is strictly greater than the break-even price, indicating that market equilibrium in an uncoordinated access setting can be fundamentally different as it opens up the possibility of providers sharing the market at higher prices.
\end{abstract}

\section{INTRODUCTION}

Recent initiatives by government agencies extend the reach of spectrum management policies that license holders (e.g., network providers) are entitled to pursue [3,6,9-11,27]. In particular, the Federal Communications Commission (FCC) introduced a new spectrum access policy model known as Private Commons to support fast time-scale spectrum transactions $[1,8]$. Under this model, ownership of spectrum remains with the license holder providing service to its primary users, but this provider may also provide spectrum access to secondary users for a fee.

As pointed out in FCC's report on secondary spectrum markets, control of secondary access in private commons can be implemented in several different ways [1]. In one possible implementation identified in [8], access to the spectrum by secondary users may be coordinated by the provider, via signals that determine when or how such access is allowed. A notable coordinated policy is the so-called threshold (reservation) policy, whereby secondary spectrum access is permitted as long as the number of channels occupied in a given spectrum

A preliminary version of this paper appeared in [20] band is below a certain threshold. Theoretical properties of the threshold policy, including optimality in certain settings, have been extensively studied in the literature ( $c f$. [22, 29-31, 34] and references therein). Access to a band may also possibly be uncoordinated, in which case primary and secondary users share access to the band on an equal basis, in a way similar to ISM bands [8].

Since cellular networks are generally over-provisioned to cope with short-term spikes, it might be possible to increase spectrum utilization through private commons. Studies indeed indicate that the majority of base stations in heavily populated areas, such as city centers, remain under-loaded at all times, suggesting that providing secondary services on licensed spectrum might increase operating revenues [2, 28].

Realizing the potential of private commons entails a number of challenges for a provider. One such challenge concerns pricing of secondary spectrum access in the face of uncertainty of demand response to the advertised price: Providing secondary access at a price returns an immediate revenue for the provider, but it also incurs an opportunity cost due to lost primary revenue as spectrum is fundamentally a finite resource. The balance between these two effects determines profitability of secondary spectrum provision, and it may possibly depend not only on the secondary price but also on the secondary demand. The relationship between secondary price and demand, also known as the demand function, is difficult to characterize explicitly, however, and may also be time-varying.

This issue is further aggravated in competitive situations in which multiple network providers compete for the same pool of secondary demand. In such situations, a provider may opt to beat the price of competitors, thereby winning the entire secondary market, or may opt to match competitors' price thereby serving part of the market at a higher price. It is not readily clear which alternative is favorable, especially under the alluded uncertainty in the price-demand relationship.

In this paper we seek to analyze and underline the differences between the outcomes of a price competition between multiple providers implementing coordinated and uncoordinated access policies in private commons, as illustrated in Fig 1(a). We consider a game theoretic setting and identify equilibrium outcomes in term of Nash equilibria. In revenue calculations, we adopt a model that explicitly captures the random nature of spectrum access requests of both primary and secondary users.

Our first contribution is to characterize and then prove the 


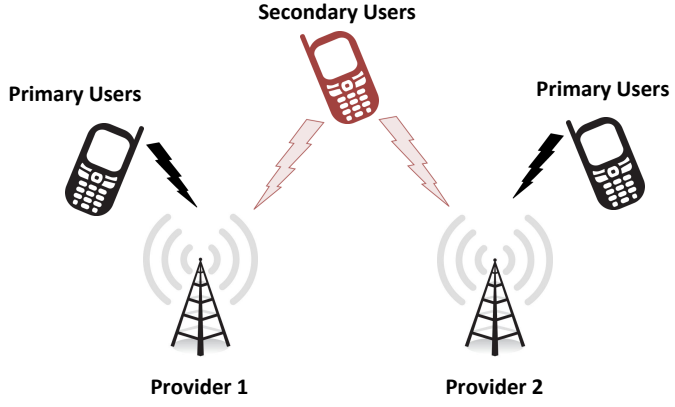

(a) Illustration of provider competition over the secondary users in a private commons setting

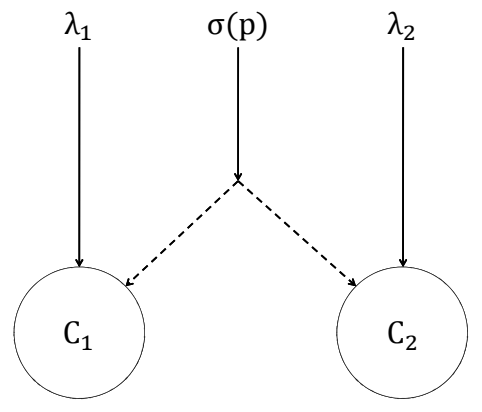

(b) Two providers each with a capacity $C_{i}, i=1,2$, compete for secondary demand $\sigma(p)$ while also serving their respective dedicated (primary) demand $\lambda_{i}$.

Fig. 1: An illustration and an abstraction of the market model considered.

existence of a break-even price for each provider under an optimal coordinated access policy. This break-even price is the lower limit to the price values for which a provider's profitability is guaranteed. The paper explicitly characterizes the break-even price, which is independent of the parameters of other providers and possesses the fundamental property of being insensitive to the demand function of secondary users. The analysis further reveals that the break-even price directly relates to the fraction of lost primary users (in the absence of secondary users), which can be expressed using the wellstudied Erlang-B function. The break-even price thus inherits all the mathematical properties of this function.

Our next contribution is to show that market sharing between providers can not be an equilibrium outcome under optimal coordinated access. We prove this claim by deriving the best response of each provider, where we show that a provider always opts to underbid its competitors, leading to a price war. The proof hinges on structural properties of the revenue function that hold irrespective of the specific relation between the price and the secondary demand. We formally establish the strictly dominating strategy of each provider and list all the possible market outcomes (i.e., Nash equilibria), which this price war can lead to. We demonstrate that the provider with the lowest break-even price wins the market. Depending on the elasticity of the secondary demand, the winner's equilibrium price may be significantly lower than the break-even prices of the competitors'. In contrast to the breakeven price, this equilibrium price cannot be identified without explicit knowledge of the secondary demand function. We note that if multiple providers share the same break-even price, they are coerced into an equilibrium in which no provider makes a profit.

As our last contribution, we show that market equilibria under uncoordinated secondary access may be drastically different than those under coordinated access. We introduce another price threshold, the market sharing price $p^{M S}$, that determines a provider's incentive to share the market. We prove that the break-even price is lower than the market shar- ing price under uncoordinated access and inelastic demand, thereby establishing the existence of a price interval on which a provider is both profitable and willing to share the market.

The rest of the paper is organized as follows. In Section II, we survey previous work. In Section III, we present the network model used to conduct our analysis. In Section IV, we analyze profitability conditions, establish market dynamics and competition outcomes under an optimal coordinated access policy. We compare and contrast these results to the uncoordinated access case and provide an analysis in Section V. We conclude the paper in Section VI.

\section{RELATED WORK}

In this section, we briefly survey related work on competition and spectrum pricing for secondary markets and highlight the differing contributions of our paper.

Network providers in spectrum markets may face competition at two different levels. The first level consists of competition between secondary network providers to lease spectrum from a primary provider (or the government) that holds a spectrum license. The second level of competition is between network providers holding a spectrum license or lease and competing to offer their services to end-users.

Many papers in the literature consider the first level of competition, while our paper addresses the second one. For instance, in the works by Jagannathan et. al.[15], Kasbekar and Sarkar [19], Duan et. al. [12], Ren et. al. [35], Niyato and Hossain [32], Sengupta and Chatterjee [36] and Xing et. al. [39], game theoretic approaches to spectrum auctioning and leasing are analyzed. The set-up of all these papers (i.e., competition between providers to lease spectrum) is different from ours (i.e., competition between providers to lure users).

Several papers study the problem of ensuring profitability in secondary spectrum markets. Niyato and Hossain [32] derive market equilibria pricing by taking into consideration the demand and supply dynamics of spectrum auctions. However, the model uses a very specific secondary demand based on the utility from owning the spectrum and how much it costs 
to lease the spectrum. Moreover, secondary users have the option to lease parts of their spectrum from different spectrum owners. On the end-user side, Alanyali et. al. $[4,5]$ establish a pricing policy which guarantees profitability for the network provider as long as a demand is generated. However, these papers assume a monopolistic framework, while ours considers an competitive oligopoly. Furthermore, $[4,5]$ consider a multicell setting with a single frequency band in each cell, while our paper focuses on an isolated cell offering multiple frequency bands.

Mutlu et al. [30] also consider a monopolistic framework and derive an optimal coordinated access policy under which revenue from secondary users is maximized. The results of that paper show that a threshold policy is optimal for coordinated access in an isolated cell, assuming that a provider advertises a fixed price (i.e., the price does not depend on the instantaneous channel occupancy).

In Ileri et al.'s work [14], a comprehensive model including both the auction and the end-user sides of the competition is studied. Different from our paper, this model focuses on the auctioning side of the competition where the revenue generated by secondary users is used to compensate for the costs of auctioning. In our model, we assume that providers own spectrum or have already leased it for a certain amount of time and need only to consider the revenue brought in by the primary and secondary users.

The works by Maille and Tuffin [25] and Maille et al. [26] use a model where both the auction side and the service side of the competition are considered. The work in [25] specifically focuses on the competition between two different but substitute technologies while [26] models a three level competition, where spectrum owners, lessees and users each make their own separate decisions. These decisions include the use of different technologies. In our model, we assume that providers offer the same type of services and therefore cannot influence the secondary users' preferences beside the price advertised. A related work by Ren et al. [35] studies and compare the market outcome achieved by respectively enforcing cooperation or competition among providers. While such external interventions might be useful in analyzing hypothetical outcomes, our model refrains from such enforcements as it aims to characterize a natural competition. In a work by Kim et al. [21], competition between two providers is analyzed where network preemption allows for primary users to evict secondary users from the system. Unlike our paper, the network model is not a finite capacity multichannel network but rather a spatial distribution of channels that turn on and off, and the analysis relies on an approximation.

None of the previous work surveyed here considers competition among network providers implementing optimal coordinated access and facing secondary demand governed by a general demand function. The characterization of the market equilibrium and demonstration of a price war won by the provider(s) with the lowest break-even price as well as the possibility of market sharing equilibria under uncoordinated access policies are unique contributions of our paper.

\section{Network \& Market Model}

In this section we introduce the network and market models considered and the accompanying notation. For convenience of exposition we present here a model with two providers, and later extend it to an arbitrary number of competing providers: Each provider $i=1,2$ has a finite number of channels $C_{i}$, and a dedicated primary-user base whose traffic generation rate (i.e., the average number of requests per unit time) is represented with $\lambda_{i}>0$. For each primary user serviced, provider $i$ collects a reward of $K_{i}$ units.

The providers compete for an additional secondary demand, which is raised through offering secondary service at a fixed access price for the duration of a contract period. The contract period is long enough (relative to inter-arrival and holding times of calls) to allow an equilibrium analysis. In the course of the contract period, neither the pricing nor the users preferences change.

If provider $i$ charges $p_{i}$ units per secondary access then the intensity of secondary demand is $\sigma\left(p_{i}\right)$. Here $\sigma(\cdot)$ is the wellknown demand function and it is assumed to be continuous and non-increasing. We denote the maximum value of the secondary demand by $\sigma_{\max }=\sigma(0)$.

We shall assume that each demand type (primary and secondary) consists of a random sequence of request arrivals that occur according to independent Poisson processes. We also assume that, if granted, each request holds a single channel for a random duration that is generally distributed with unit mean, independently of other requests and arrival times. We shall assume that the channel holding statistics are identical for primary and secondary requests. Such an assumption is valid when both types of traffic are generated by similar applications.

The general form of aggregate secondary demand $\sigma(p)$ captures the heterogeneity of customer preferences. Indeed, the demand function implicitly represents the fraction of users (user types) that find each price value acceptable. The generality of the demand function allows consideration of different user types. The separation between primary and secondary users and the random nature of service times capture additional levels of heterogeneity in our model.

Secondary demand is assumed to be attracted to the provider charging the lowest price. This behavior can be explained by price aversion, a concept employed in marketing management [38]. When both providers charge the same price, the resulting secondary demand splits between the two providers according to a static probability vector $\left[\alpha_{1}, \alpha_{2}\right]$ such that $\alpha_{1}+\alpha_{2}=1$ and $\alpha_{1}, \alpha_{2}>0$. Namely, each provider $i$ receives a secondary demand of volume $\alpha_{i} \sigma\left(p_{i}\right)$ every time market prices are equal.

Each provider $i$ also has the choice of admitting or rejecting secondary requests according to an access policy, which we denote by $A_{i}$. We assume that actions taken by $A_{i}$ depend only on the number of each class of users (primary and secondary) in the system. Thus, $A_{i}$ belongs to the class of occupancybased policies, the performance of which are insensitive to the call length distribution except through the mean [31]. Hence, without loss of generality, we can assume exponentially distributed service times for the purpose of analysis in the rest of this paper. 
Since providers have a finite number of channels to provide service with, they cannot accommodate new requests if all of the channels are occupied. This results in some requests being blocked. We define $B_{i, j}\left(\lambda_{i}, \sigma, A_{i}\right)$ as the blocking probability for class $j$ users ( $j=1$ for primary and 2 for secondary) when secondary demand is $\sigma$ and the access policy is $A_{i}$.

The goal of each provider is to maximize the total revenue collected. The revenue rate of provider $i$ when it services secondary demand of $\sigma$ units is given by:

$$
\begin{array}{r}
W_{i}\left(p_{i}, \sigma, A_{i}\right)=\left(1-B_{i, 2}\left(\lambda_{i}, \sigma, A_{i}\right)\right) \sigma p_{i} \\
+\left(1-B_{i, 1}\left(\lambda_{i}, \sigma, A_{i}\right)\right) \lambda_{i} K_{i} .
\end{array}
$$

Here the first and the second terms are respectively the revenue generated by primary and secondary requests that are admitted by the provider. Each term represents the expected long time rates per unit time.

Since the secondary demand a provider receives depends on prices of both providers, so does the revenue of the provider. We define the reward $R_{i}\left(p_{i}, p_{-i}\right)$ of provider $i$ as its revenue when provider $i$ and its competitor $-i$ charge secondary access $p_{i}$ and $p_{-i}$ units respectively. Namely,

$$
R_{i}\left(p_{i}, p_{-i}\right)= \begin{cases}W_{i}\left(p_{i}, \sigma\left(p_{i}\right), A_{i}\right) & \text { if } p_{i}<p_{-i} \\ W_{i}\left(p_{i}, \alpha_{i} \sigma\left(p_{i}\right), A_{i}\right) & \text { if } p_{i}=p_{-i} \\ W_{i}\left(p_{i}, 0, A_{i}\right) & \text { if } p_{i}>p_{-i} .\end{cases}
$$

Hence the reward is affected by the amount of secondary demand provider $i$ captures through the relationship between its own price $p_{i}$ and the price of the other provider $p_{-i}$. Once the prices determine the secondary demand for each provider, the rewards are further shaped by the providers' access policies. Each provider has full information on its own network parameters and can observe the prices advertised by its competitors.

\section{Optimal Coordinated Access Policy}

\section{A. Profitability}

For a given secondary demand $\sigma$ and secondary price $p$, let $A^{*}(p, \sigma)$ denote a coordinated access policy that maximizes the revenue rate for a provider (for analyses in which we consider a single provider, we will drop index $i$ from our notation for the sake of simplicity). We refer to $A^{*}(p, \sigma)$ as the optimal coordinated access policy. We represent the resulting maximal revenue $W^{*}(p, \sigma)$ as follows:

$$
W^{*}(p, \sigma)=W\left(p, \sigma, A^{*}(p, \sigma)\right)=\max _{A} W(p, \sigma, A) .
$$

One can formulate the provider's optimization problem using a Markov decision process (MDP), where the state is the total number of users in the network. Note that primary and secondary users have identical channel holding statistics, hence once admitted to the network they are indistinguishable. At every state, the provider needs to make a decision whether to admit or reject a secondary user arrival in order to maximize its expected revenue. MDPs can be solved with dynamic programming (DP) techniques [7]. Under the given assumptions, it is well-known that the coordinated access policy that yields the optimal solution to our DP problem is a threshold (reservation) policy: Secondary users are admitted by a provider when the channel occupancy of the provider is below a threshold $T \geq 0$ and they are blocked otherwise [22, 29,31,34]. The optimal threshold value depends on all parameters of the provider including intensity of the secondary demand. We let the notation $A=T$ correspond to the implementation of a threshold policy with the specific threshold value being equal to $T$.

In the competitive setting considered in this paper it will be important to identify conditions under which an optimal policy $A^{*}(p, \sigma)$ ever accepts a secondary request. Under such conditions the secondary price-demand pair $(p, \sigma)$ yields profit relative to serving primary demand only; in turn $(p, \sigma)$ represents an economically viable situation for a provider. The issue is closely related with the opportunity cost of accepting a secondary request: On the one hand such a request brings an immediate revenue of $p$, on the other hand it may cause rejecting future requests, possibly with higher immediate revenue, due to the channel that it holds temporally. To identify the profitability of admitting a secondary user, we utilize a policy improvement technique based on $[4,22]$. Specifically, we identify a price condition for which there exists a policy that yields a better revenue than a policy that flatly rejects all secondary arrivals. This determines the sign of the balance in the trade-off when making a control decision to admit a secondary user or not. We state our main result on this profitability condition in the following theorem:

Theorem IV.1 For $\sigma>0$ there exists a break-even price $p^{B E}$ given by:

$$
p^{B E}=K E(\lambda, C),
$$

where $E(\lambda, C)=\frac{\lambda^{C} / C !}{\sum_{k=0}^{C} \lambda^{k} / k !}$ is the Erlang-B formula. such that:

(a) $W^{*}(p, \sigma)>W^{*}(p, 0)$ if $p>p^{B E}$,

(b) $W^{*}(p, \sigma)=W^{*}(p, 0)$ if $p \leq p^{B E}$.

Proof: In order to calculate for which prices it is profitable to admit secondary users, we model the optimization problem as an MDP. Thus, we set up an infinite horizon average cost dynamic programming problem and identify the prices at which the optimal policy allows for the admission of secondary users into the network at some states. To do so, we take the total number of users in the network (i.e., occupancy) denoted by $y$ as the state of the system, $\bar{J}$ as the time-average reward and $h(y)$ as the differential reward function [7]. $\bar{J}$ can be interpreted as the average reward collected from incoming arrivals over a period of time which length goes to infinity, whereas the differential reward function $h(y)$ characterizes the expected difference when we start the process from a particular state $y$ instead of an arbitrary state $y^{\prime}$ which we take as the reference such that $h\left(y^{\prime}\right)=0$. In our case, and without any loss of generality, we set $y^{\prime}=0$.

We uniformize the process with the maximum possible transition rate out of any state, which we denote by $\nu \triangleq$ $\lambda+\sigma+C$. Since the service rate is the same for both primary and secondary users, they are indistinguishable once in the system. Following this observation, at state $\{y: 0 \leq y \leq C\}$ 
a user (either primary or secondary) will leave the system with probability $\frac{y}{\nu}$. With probability $\frac{\lambda}{\nu}$ a primary user will arrive, with probability $\frac{\sigma}{\nu}$ a secondary user will arrive, and with probability $\frac{C-y}{\nu}$ the state will remain the same (i.e., nothing happens). Note that an arrival of either kind to a full network is not admitted and thus no reward is collected. Then the well established Bellman equations for the average reward problem can be formulated as follows:

$$
\begin{aligned}
& \bar{J}+h^{*}(y)=\frac{1}{\nu}\left\{y h^{*}(y-1)+(C-y) h^{*}(y)\right. \\
& +\lambda\left(K+h^{*}(y+1)\right) \\
& \left.+\sigma \max \left(p+h^{*}(y+1), h^{*}(y)\right)\right\},
\end{aligned}
$$

for $0<y \leq C-1$. The last term on the right hand side of the equation reflects the admission choice to be made, that is either admit an incoming secondary user and collect a reward of $p$ while increment the state or reject the arrival and preserve the state.

We also consider the two special cases, first when the network is full:

$$
\bar{J}+h^{*}(C)=\frac{1}{\nu}\left\{C h^{*}(C-1)+(\lambda+\sigma) h^{*}(C)\right\},
$$

and next when the network is empty:

$$
\begin{aligned}
\bar{J}+h^{*}(0) & =\frac{1}{\nu}\left\{C h^{*}(0)+\lambda\left(K+h^{*}(1)\right)\right. \\
& \left.+\sigma \max \left(p+h^{*}(1), h^{*}(0)\right)\right\} .
\end{aligned}
$$

Let us define the lock-out policy as an access policy where all secondary users are rejected, regardless of network occupancy. We will approach this pricing decision problem by determining when the lock-out policy on secondary users stops being optimal. Assuming a lock-out policy, which we denote by the use of the superscript $L O$, Eq. (5) reduces to:

$$
\begin{aligned}
& \bar{J}+h^{L O}(y)=\frac{1}{\nu}\left\{y h^{L O}(y-1)+(C-y) h^{L O}(y)\right. \\
& \left.+\lambda\left(K+h^{L O}(y+1)\right)+\sigma h^{L O}(y)\right\} .
\end{aligned}
$$

From the last argument of Eq. (5), it is clear that when the state of the network is $y$, a lock-out policy is optimal if and only if $\max \left(p+h^{L O}(y+1), h^{L O}(y)\right)=h^{L O}(y)$ or:

$$
p \leq h^{L O}(y)-h^{L O}(y+1) .
$$

Therefore if $p \geq h^{L O}(y)-h^{L O}(y+1)$, a lock-out policy is no longer optimal, which is equivalent to starting to admit some secondary users. We shall next obtain an analytical expression of the quantity $H(y) \triangleq h^{L O}(y)-h^{L O}(y+1)$.

Writing Eq. (6) for every state $y$ and taking the difference between every two consecutive states yields the following set of equations:

$$
\begin{array}{ccc}
(\lambda+1) H(0) & = & \lambda H(1) \\
\vdots & \vdots & \vdots \\
(\lambda+y) H(y-1) & = & \lambda H(y)+(y-1) H(y-2) \\
\vdots & \vdots & \vdots \\
(\lambda+C) H(C-1) & = & \lambda K+(C-1) H(C-2)
\end{array}
$$

The solution to this set of equations is

$$
H(y)=K \frac{E(\lambda, C)}{E(\lambda, y)} \quad \text { for } 0 \leq y \leq C-1 .
$$

Since we are specifically interested in finding the price $p$ at which it is optimal to admit at least one secondary user into the network, through Eq. (7), we know that this price must be greater than or equal to

$$
\min _{0 \leq y \leq C-1} H(y)=\min _{0 \leq y \leq C-1}\left(h^{L O}(y)-h^{L O}(y+1)\right) .
$$

By observing how Eq. (8) changes with respect to $y$, one can come to the conclusion that $H(y)$ is increasing in $y$, the minimum value such a price $p$ can take is:

$$
p^{B E} \triangleq H(0)=K \frac{E(\lambda, C)}{E(\lambda, 0)}=K E(\lambda, C) .
$$

Therefore, as long as the price is greater than $p^{B E}$, there exists at least one state $y$ (i.e., when the system is empty) at which admitting secondary customers yields a better revenue rate than the revenue rate under a lock-out policy.

Theorem IV.1(a) states that if the price exceeds $p^{B E}$ then serving secondary demand yields strictly higher revenue for a provider than not serving it. Conversely, part (b) of the theorem states that secondary demand does not lead to any revenue improvement otherwise, implying that rejecting the entire secondary demand is optimal for such prices. In effect, at $p^{B E}$ the immediate revenue balances the opportunity cost of a secondary request. We therefore coin $p^{B E}$ as the break-even price of a provider.

It is striking that the break-even price expression (9) does not depend on the secondary demand. Namely, any price above $p^{B E}$ strictly improves the revenue of a provider regardless of how much secondary demand it generates. This result can be intuitively understood as follows: The size of secondary demand does not play a role in profitability, for any positive secondary demand can be thinned down arbitrarily by the coordinated access policy. We have shown that at the breakeven price the lock-out policy stops being optimal, which is equivalent to stating that secondary access is profitable when the network is empty. Since the profitability of the first admitted secondary user depends on a network where there are no other secondary users, secondary demand does not affect the break-even price.

Figures 2(a) and 2(b) illustrate how the normalized breakeven price (i.e., $p^{B E} / K$ ) changes with respect to relevant network parameters, namely the system capacity $C$ and the network load $\lambda / C$. The normalized price is given by the Erlang-B function, which has been well studied in teletraffic theory. In particular upper and lower bounds are obtained in $[13,17]$, and it is demonstrated in [16] that for a given arrival load $\lambda$, the Erlang-B function (hence, the break-even price) is a convex function of the capacity $C$, as can be observed from Figure 2(a). It is also worth noting that as the network capacity increases, the value of the break-even price at the critical load where $\lambda=C$ decreases as demonstrated in Figure 2(b).

Figure 2(b) shows that for an over-provisioned network (in which primary load $\lambda$ is below the capacity $C$ by a 


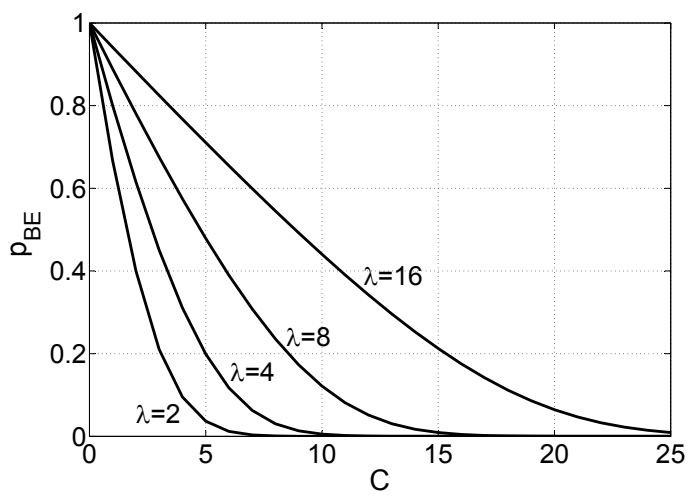

(a) Break-even price $p^{B E}$ with respect to network capacity $C$ for different primary arrival rates $\lambda$ and primary price $K=1$.

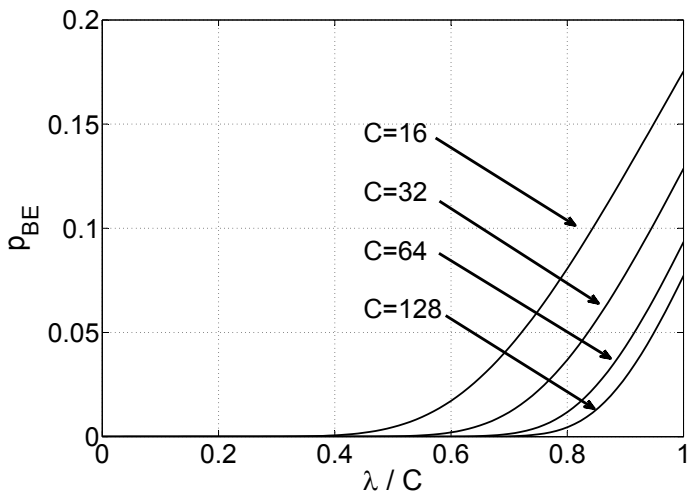

(b) Break-even price $p^{B E}$ with respect to network load $\lambda / C$ for different network capacities $C$ and primary price $K=1$.

Fig. 2: Behavior of break-even price as a function of network parameters.

significant margin), the break-even price is substantially lower than the primary price. We observe that for $C=16$ the normalized break-even price is negligible compared to the primary price for network loads below $40 \%$, a number close to the network utilization measurements reported in [2]. As the network capacity increases, it takes even higher network loads to observe the slightest increase in the break-even price, almost as high as $80 \%$ when the capacity is increased to $C=128$. This result suggests that, in an over-provisioned network, spectrum sharing at secondary prices that are low relative to primary reward would result in net profit, regardless of the secondary demand.

\section{B. Best Response}

In competing for and serving secondary demand, a provider's action consists of an advertised price for secondary access and an access policy to coordinate secondary access. For any price, and for any demand the price raises, each provider's revenue is highest under optimal coordination. Hence optimal coordination is a dominating choice uniformly for all situations. In this section we will assume all providers implement optimal coordinated access. With this assumption each provider's strategic action reduces to a pricing decision.

Before we move to establish market equilibrium, it is beneficial to first establish the strictly dominated strategies for both providers under optimal coordinated access. This allows for the characterization of a provider's best response strategy for any price its competitor chooses. In the next theorem, we state that the best response of a provider is to set its price slightly lower than the competition in order to capture all of the secondary demand rather than sharing the secondary demand at that price. This can be formalized as follows:

Theorem IV.2 If $p>p^{B E}$, for any given $\alpha \in[0,1]$ there exists a price $p^{\prime} \in\left(p^{B E}, p\right)$ such that:

$$
W^{*}\left(p^{\prime}, \sigma\left(p^{\prime}\right)\right)>W^{*}(p, \alpha \sigma(p)) .
$$

Before we prove Theorem IV.2, it is beneficial to establish the strictly dominated strategies for both providers under optimal coordinated access. This allows for the characterization of provider $i$ 's best response strategy for any price it's competitor chooses. To do so we introduce two lemmas. In the first lemma for two given secondary demand values of $\sigma_{1}$ and $\sigma_{2}$ such that $\sigma_{1}>\sigma_{2}$, we will demonstrate that the revenue rate when facing higher secondary demand $\sigma_{1}$ is never less than the revenue rate when facing lower secondary demand $\sigma_{2}$ (i.e., $\left.W^{*}\left(p_{i}, \sigma_{1}\right) \geq W^{*}\left(p_{i}, \sigma_{2}\right)\right)$.

Lemma IV.1 Let $p>0$. For any $\sigma_{1}, \sigma_{2}$ such that $\sigma_{1}>\sigma_{2}$ :

$$
W^{*}\left(p, \sigma_{1}\right) \geq W^{*}\left(p, \sigma_{2}\right) .
$$

Proof. Consider the optimal access policy $A^{*}\left(p, \sigma_{2}\right)$ which yields a revenue rate of $W^{*}\left(p, \sigma_{2}\right)$ for demand $\sigma_{2}$. Now consider a policy $\hat{A}\left(p, \sigma_{1}\right)$ for demand $\sigma_{1}$, which does a random thinning of the secondary demand and brings it to $\sigma_{2}$ (i.e., $\hat{A}\left(p, \sigma_{1}\right)$ accepts each arrival with probability $\sigma_{2} / \sigma_{1}$. Note that the thinned arrival process is still Poisson [22]. Once the secondary demand is reduced, access policy $A^{*}\left(p, \sigma_{2}\right)$ is implemented. Hence $\hat{A}\left(p, \sigma_{1}\right)$ and $A^{*}\left(p, \sigma_{2}\right)$ generate the same revenue rate, i.e., $W\left(p, \sigma_{1}, \hat{A}\right)=W^{*}\left(p, \sigma_{2}\right)$.

Since by definition $A^{*}\left(p, \sigma_{1}\right)$ is the optimal coordinated access policy when secondary demand is $\sigma_{1}$, we know that it does not generate a revenue less than the revenue generated by the policy we have just described. We can formulate this conclusion as:

$$
W^{*}\left(p, \sigma_{1}\right) \geq W\left(p, \sigma_{1}, \hat{A}\right)=W^{*}\left(p, \sigma_{2}\right) .
$$

In the previous lemma, we have demonstrated that an increase in secondary demand does not result in a decrease in the revenue rate of a provider. In the second lemma we will build on the previous lemma to show that when the price is set above the break-even price, an increase in secondary demand translates into strict increase in the revenue rate. 


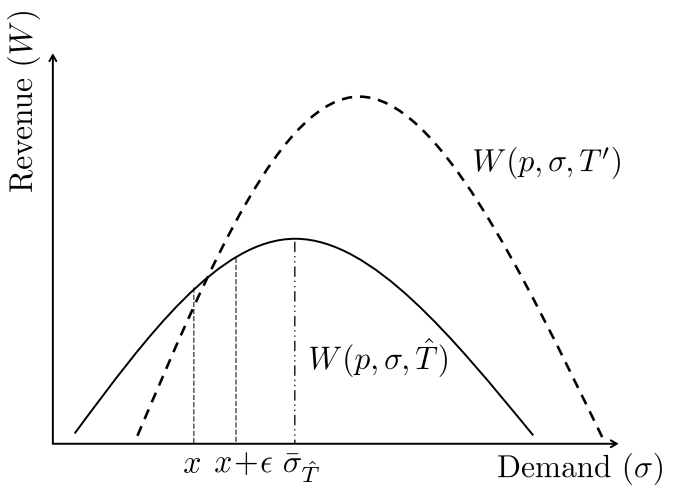

(a) Case 1: $\bar{\sigma}_{\hat{T}} \notin[x, x+\epsilon)$

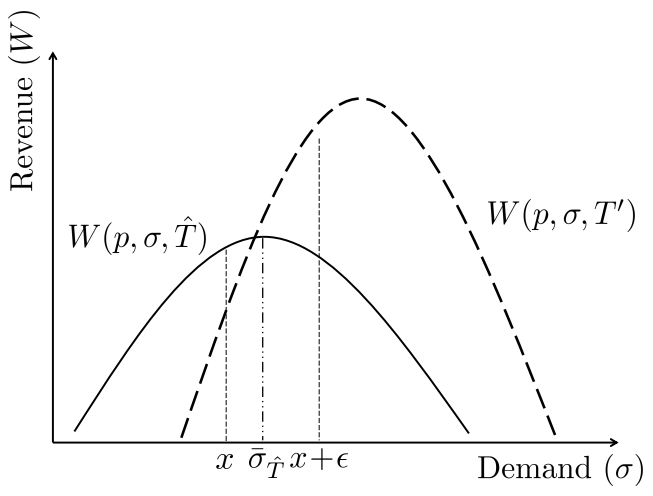

(b) Case 2: $\bar{\sigma}_{\hat{T}} \in[x, x+\epsilon)$

Fig. 3: Illustration of the two cases considered in the proof of Lemma IV.2

Lemma IV.2 Let $p>p^{B E}$. For any $\sigma_{1}, \sigma_{2}$ such that $\sigma_{1}>\sigma_{2}$ : $W^{*}\left(p, \sigma_{1}\right)>W^{*}\left(p, \sigma_{2}\right)$.

Proof: We know that when the price is greater than the break-even price (i.e., $p>p^{B E}$ ), an optimal admission policy will never choose the threshold value $T=0$. Since in this lemma we only consider such prices, we can formalize this result through the formulation

$$
\max _{0 \leq T \leq C} W(p, \sigma, T)=\max _{1 \leq T \leq C} W(p, \sigma, T) .
$$

In Mutlu et al.'s work [30], it is shown that for a fixed admission threshold value $T>0, W(p, \sigma, T)$ is a unimodal function with respect to $\sigma$ for any $p$. In other words, $W(p, \sigma, T)$ is strictly increasing until is reaches a certain maximum and strictly decreasing afterwards. We define the value of $\sigma$ at which $W(p, \sigma, T)$ attains its maximum value over the interval $\left[0, \sigma_{\max }\right]$ for an admission control policy with fixed threshold $T$ by:

$$
\bar{\sigma}_{T}=\underset{\sigma \in\left[0, \sigma_{\max }\right]}{\operatorname{argmax}} W(p, \sigma, T) .
$$

We define $d$ to be the minimum of the distances between any two distinct maxima of $W(p, \sigma, T)$ for different values of $T$ so that

$$
d=\inf _{m, n \in 1,2, \ldots, C}\left|\bar{\sigma}_{m}-\bar{\sigma}_{n}\right|, \quad \bar{\sigma}_{m} \neq \bar{\sigma}_{n} .
$$

Since there are a finite number of possible threshold policies, the infimum is achieved (i.e., inf $=\min$ ) and $d>0$. Having defined the minimum distance between distinct maxima of two different threshold revenue functions, we will prove the lemma by first showing that

$$
W^{*}(p, x+\epsilon)>W^{*}(p, x), \quad \forall x \in\left[0, \sigma_{\max }\right] \text { and } \epsilon<d,
$$

where $\epsilon$ is a constant the value of which does not depend on the secondary demand $x$.

It should be noted that the value of $\epsilon$ does not depend on $x$. From the way $\epsilon$ has been chosen, there can be at most one distinct maximum over the interval $[x, x+\epsilon]$. In the rest of this proof, let $\hat{T}$ denote the optimal threshold value at $x$ (if there are more than one we can choose any). We will complete our proof by distinguishing between two cases, as illustrated in Fig. 3:

Case 1: $\bar{\sigma}_{\hat{T}} \notin[x, x+\epsilon)$.

Given the unimodality of $W(p, \sigma, \hat{T})$, this function must be either decreasing or increasing with respect to $\sigma$ in the interval $[x, x+\epsilon]$. Furthermore, it must also be true that $x<$ $\bar{\sigma}_{\hat{T}}$. Otherwise, if $x \geq \bar{\sigma}_{\hat{T}}$, through the way we have defined $\bar{\sigma}_{\hat{T}}$ in Eq. (12) we would have $W^{*}(p, x)=W(p, x, \hat{T})<$ $W\left(p, \bar{\sigma}_{\hat{T}}, \hat{T}\right)$, which is a contradiction to Lemma IV.1, which we can rewrite in the following form:

$$
W^{*}(p, x) \geq W\left(p, \bar{\sigma}_{\hat{T}}, \hat{T}\right), \quad \forall x \geq \bar{\sigma}_{\hat{T}} .
$$

Thus $W(p, \sigma, \hat{T})$ cannot be decreasing but must be increasing in $\sigma$ over the interval $[x, x+\epsilon)$. By definition of optimality:

$$
W^{*}(p, x+\epsilon) \geq W(p, x+\epsilon, \hat{T})>W(p, x, \hat{T})=W^{*}(p, x) .
$$

Case 2: $\bar{\sigma}_{\hat{T}} \in[x, x+\epsilon)$.

$W(p, \sigma, \hat{T})$ attains its maximum value over $[x, x+\epsilon)$ at $\bar{\sigma}_{\hat{T}}$. Given the unimodality of $W(p, \sigma, \hat{T})$ with respect to $\sigma$, the revenue function must be increasing on the interval $\left[x, \bar{\sigma}_{\hat{T}}\right]$. Next we show that the revenue must remain increasing over $\left[\bar{\sigma}_{\hat{T}}, x+\epsilon\right)$ for at least one other fixed threshold policy, which we prove by contradiction. Suppose that at $\sigma=\bar{\sigma}_{\hat{T}}$ there exists no threshold policy under which the revenue rate is both increasing and greater than or equal to $W(p, \sigma, \hat{T})$. Then, the revenue function under the optimal policy must be decreasing right after $\bar{\sigma}_{\hat{T}}$ as it is continuous in $\sigma$ (see also proof of Theorem IV.2). This contradicts Lemma IV.1. Hence, there must exist at least one other threshold policy $A=T^{\prime}$ such that the revenue rate under this new threshold value $W\left(p, \bar{\sigma}_{\hat{T}}, T^{\prime}\right)$ is increasing and satisfies $W\left(p, \bar{\sigma}_{\hat{T}}, T^{\prime}\right) \geq W\left(p, \bar{\sigma}_{\hat{T}}, \hat{T}\right)$. Since the interval $[x, x+\epsilon)$ contains at most one distinct maximum, $W\left(p, x, T^{\prime}\right)$ must remain increasing over the interval $\left[\bar{\sigma}_{\hat{T}}, x+\epsilon\right)$. Then we can conclude

$$
\begin{array}{r}
W^{*}(p, x+\epsilon) \geq W\left(p, x+\epsilon, T^{\prime}\right)>W\left(p, \bar{\sigma}_{\hat{T}}, T^{\prime}\right) \\
\geq W\left(p, \bar{\sigma}_{\hat{T}}, \hat{T}\right)>W(p, x, \hat{T})=W^{*}(p, x) .
\end{array}
$$

Having shown that $W^{*}(p, x+\epsilon)>W^{*}(p, x)$ for $\epsilon<d$ under both cases, we can finally proceed with making the 
connection between our proof and the lemma by first stating:

$$
\begin{gathered}
W^{*}\left(p, \sigma_{1}\right)>W^{*}\left(p, \sigma_{1}-\epsilon\right)>W^{*}\left(p, \sigma_{1}-2 \epsilon\right)> \\
\ldots>W^{*}\left(p, \sigma_{1}-k \epsilon\right),
\end{gathered}
$$

where $k$ is the largest integer such that $\sigma_{1}-k \epsilon>\sigma_{2}$. Since we can take any $\epsilon<d$, we can choose one final $\epsilon^{\prime}=\sigma_{1}$ $k \epsilon-\sigma_{2}<d$, such that:

$$
W^{*}\left(p, \sigma_{1}-k \epsilon\right)=W^{*}\left(p, \sigma_{2}+\epsilon^{\prime}\right)>W^{*}\left(p, \sigma_{2}\right) .
$$

Combining Eqs. (13) and (14) we get

$$
W^{*}\left(p, \sigma_{1}\right)>W^{*}\left(p, \sigma_{2}\right) \text {. }
$$

Proceeding with the proof of our theorem, we show that as long as the price is lowered by less than a certain amount, the relationship established in the Lemma IV. 2 can be extended to different prices such that $W^{*}\left(p^{\prime}, \sigma\left(p^{\prime}\right)\right)>W^{*}(p, \alpha \sigma(p))$ where $p^{\prime}<p$.

Proof of Theorem IV.2 Through Lemma IV. 2 we know that the following inequality holds:

$$
W^{*}(p, \sigma(p))>W^{*}(p, \alpha \sigma(p)) .
$$

For a fixed threshold value $T$, the revenue takes the form:

$$
\begin{aligned}
W(p, \sigma(p), T)= & \left(1-B_{2}(\lambda, \sigma(p), T)\right) \sigma(p) p \\
& +\left(1-B_{1}(\lambda, \sigma(p), T)\right) \lambda K
\end{aligned}
$$

where

$$
\begin{aligned}
& B_{1}(\lambda, \sigma(p), T)= \\
& \quad \frac{\frac{(\lambda+\sigma(p))^{T} \lambda^{C-T}}{C !}}{\sum_{n=0}^{T-1} \frac{(\lambda+\sigma(p))^{n}}{n !}+(\lambda+\sigma(p))^{T} \sum_{n=T}^{C} \frac{\lambda^{n-T}}{n !}},
\end{aligned}
$$

and

$$
\begin{aligned}
& B_{2}(\lambda, \sigma(p), T)= \\
& \quad \frac{(\lambda+\sigma(p))^{T} \sum_{n=T}^{C} \frac{\lambda^{n-T}}{n !}}{\sum_{n=0}^{T-1} \frac{(\lambda+\sigma(p))^{n}}{n !}+(\lambda+\sigma(p))^{T} \sum_{n=T}^{C} \frac{\lambda^{n-T}}{n !}},
\end{aligned}
$$

the derivation of which is given in [30]. Since the respective blocking probabilities of primary secondary users $B_{1}(\cdot)$ and $B_{2}(\cdot)$ are a function of $p$ through $\sigma(p)$, which is assumed to be continuous in $p$, we conclude from Eq. (16) that $W(p, \sigma(p), T)$ is also continuous in $p$.

From the way we have defined the optimal access policy in Eq. (3), $W^{*}(p, \sigma(p))$ is also continuous in $p$ as we consider a finite set of possible values which $T$ can take [18, pp. $11 \& 135]$.

First let us assume that there exists a $\hat{p} \in\left(p^{B E}, p\right)$ such that

$$
W^{*}(\hat{p}, \sigma(\hat{p})) \geq W^{*}(p, \sigma(p)) .
$$

Then it follows by Eq. (15) that

$$
W^{*}(\hat{p}, \sigma(\hat{p}))>W^{*}(p, \alpha \sigma(p))
$$

and $p^{\prime}$ can be set equal to $\hat{p}$. On the other hand, assume that there exists no such price $\hat{p}<p$ for which

$$
W^{*}(\hat{p}, \sigma(\hat{p})) \geq W^{*}(p, \sigma(p)) .
$$

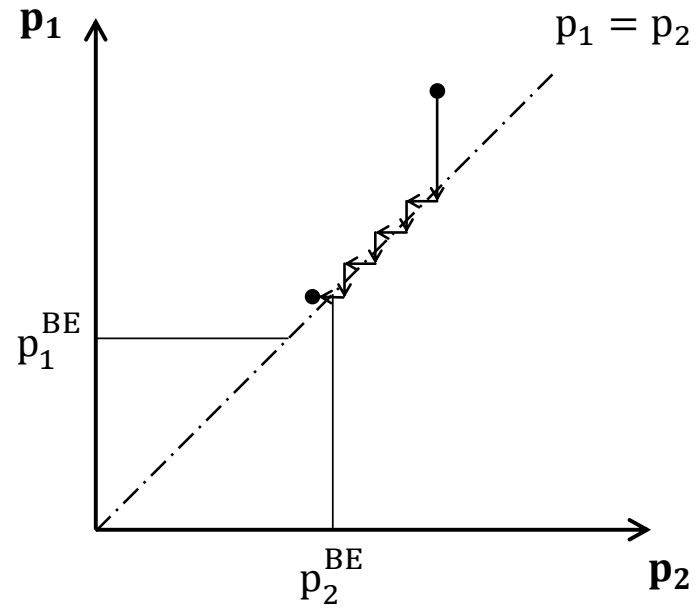

Fig. 4: Representation of a price war as a result of best response dynamics under coordinated access.

This implies that the revenue is monotonically increasing for all $\hat{p}<p$ such that:

$$
W^{*}(\hat{p}, \sigma(\hat{p}))<W^{*}(p, \sigma(p)) .
$$

Then by continuity, the following can be stated for $W^{*}(p, \sigma(p)): \forall \epsilon>0, \exists \delta(\epsilon, p)>0$ s.t. if $|p-\hat{p}|<\delta$ then

$$
\left|W^{*}(p, \sigma(p))-W^{*}(\hat{p}, \sigma(\hat{p}))\right|<\epsilon .
$$

Making use of Eq. (17) and our assumption that $\hat{p}<p$, we can remove the absolute value from the previous equation and simplify it to:

$$
W^{*}(p, \sigma(p))-W^{*}(\hat{p}, \sigma(\hat{p}))<\epsilon .
$$

Taking $\epsilon=W^{*}(p, \sigma(p))-W^{*}(p, \alpha \sigma(p))$ and cancelling the terms $W^{*}(p, \sigma(p))$ on both sides of the inequality (18) we obtain $-W^{*}(\hat{p}, \sigma(\hat{p}))<-W^{*}(p, \alpha \sigma(p))$. Multiplying both sides by -1 , the equation finally takes the form $W^{*}(\hat{p}, \sigma(\hat{p}))>$ $W^{*}(p, \alpha \sigma(p))$ and $p^{\prime}$ can be set equal to $\hat{p}$.

Theorem IV.2 states that if a provider profits at a given price, obtaining the entire secondary demand at that price is strictly more profitable than obtaining part of the demand at a slightly higher price. This property reflects an incentive for each provider to unilaterally deviate from offering the same price as its opponent, provided that the price is strictly above its break-even price. This best response dynamics is illustrated in Figure 4 and the resulting market equilibrium is formally analyzed in the next section.

\section{Market Equilibrium}

Having identified the best response of a network provider under coordinated access in Theorem IV. 2 in the previous section, we now proceed to establish the market equilibrium. Given initial prices $p_{1}$ and $p_{2}$ such that $p_{i}>p_{i}^{B E}, i=1,2$, the two providers will lower their prices in turn. This process continues until the market price drops so low that the provider with the higher break-even price finds himself unable to lower its price any further without incurring a net loss. 
We start with a characterization for competitive equilibria in the considered scenario. However, before we do that, it is important to recall the formal definition of a Nash equilibrium.

Definition IV.1 A pricing strategy profile $\left(p_{1}, p_{2}\right)$ is a Nash equilibrium for rewards $R_{i}\left(p_{1}, p_{2}\right)$ if and only if

$$
R_{1}\left(p_{1}, p_{2}\right)=\max _{p} R_{1}\left(p, p_{2}\right)
$$

and

$$
R_{2}\left(p_{1}, p_{2}\right)=\max _{p} R_{2}\left(p_{1}, p\right) .
$$

Next, we provide a theorem which identifies possible market outcomes in terms of Nash equilibria, the proof of which can be found in the appendix.

The first part of the theorem is concerned with the case when one provider (without loss of generality provider 1) has strictly lower break-even price than the other provider. In the theorem, we show that the provider with the lower break-even price captures the entire market by pricing below its competitor's break-even price. However, when the price is continuous, it is impossible to provide an exact price that achieves this best response. Hence, following a well-known approach used in game theory to address this technicality [33, pages 64-67], we assume that each provider's price is a multiple of a sufficiently small discretization step $\epsilon$.

Additionally, the exact value of the equilibrium price $p_{1}$ depends on where provider 1's revenue is maximized over the interval $\left[p_{1}^{B E}, p_{2}^{B E}-\epsilon\right]$. We formally define this maximum as the following:

$$
\bar{W}_{1}^{*}=\max _{p \in\left[p_{1}^{B E}, p_{2}^{B E}-\epsilon\right]} W_{-\epsilon}^{*}(p, \sigma(p)) .
$$

Note that the revenue may attain this maximum at several prices on the interval, which we denote by the following set:

$$
\mathcal{P}=\underset{p \in\left[p_{1}^{B E}, p_{2}^{B E}-\epsilon\right]}{\operatorname{argmax}} W_{1}^{*}(p, \sigma(p)) .
$$

The other provider is unable to underbid its competition in a profitable fashion. Hence it opts for any price that doesn't capture the secondary demand. In the equilibrium this price must also not give an incentive to the winner to deviate to a higher price. We next define the lowest price provider 2 can choose for which there exists an incentive for provider 1 to deviate from $\mathcal{P}$ :

$$
p^{\text {max }} \triangleq \underset{\hat{p} \geq p_{2}^{B E}}{\operatorname{arginf}}\left\{\max _{p \in\left[p_{1}^{B E}, \hat{p}\right]} W_{1}^{*}(p, \sigma(p))>\bar{W}_{1}^{*}\right\} .
$$

If no such price exists, then we simply set $p^{\max }=\infty$. Then this price effectively limits the price choice of provider 2 from above. Setting any price above $p^{\max }$ creates an incentive for provider 1 to deviate, thus disturbing the equilibrium. If provider 2 were to choose a price $p_{2}>p^{\max }$, then what follows is that provider 1 raises its price to this new maximizing price. However, provider 2 would then respond with underbidding provider 1 as a result of Theorem IV.2.

The second part of the theorem concerns the symmetric case when both providers have the same break-even price. In that case the unique Nash equilibrium outcome is defined by both providers charging their break-even prices, unable to capture the entire market due to profitability constraints.

\section{Theorem IV.3 (Nash Equilibria)}

(a) If $p_{1}^{B E}<p_{2}^{B E}$ then one or more Nash equilibria exist and have the strategy profile $\left(p_{1}, p_{2}\right)$ where

$$
\begin{gathered}
p_{1} \in \mathcal{P} \\
p_{2} \in\left(p_{1}, p^{\max }\right),
\end{gathered}
$$

where $\mathcal{P}$ is as given by Eq. (20) and $p^{\max }$ by Eq. (21).

(b) If $p_{1}^{B E}=p_{2}^{B E}$ then there exists a unique Nash Equilibrium given by the strategy profile $\left(p_{1}, p_{2}\right)$ such that

$$
p_{1}=p_{2}=p_{1}^{B E} .
$$

Proof: See Appendix.

The following two examples aim to illustrate that qualitative differences in the placement of Nash equilibria are governed by the secondary demand function $\sigma(p)$. These examples are based on demand functions commonly used in the economics literature that are respectively exponentially and linearly decreasing with price [37].

Example IV.1 Suppose that the secondary demand function follows a negative exponential demand $\sigma(p)=10 e^{-0.2 p}$, which indicates sufficiently low price elasticity of demand so that the revenue rate remains increasing with price. We set the network parameters for both providers as:

$$
\left(\lambda_{1}, C_{1}, K_{1}\right)=(1,2,20), \quad\left(\lambda_{2}, C_{2}, K_{2}\right)=(10,5,35),
$$

which, through Eq. (9), yield $p_{1}^{B E}=4.00, p_{2}^{B E}=19.74$. Figure 5(a) demonstrates the low-elasticity property of provider 1 's revenue rate function, $W_{1}^{*}\left(p_{1}, \sigma\left(p_{1}\right)\right)$. The revenue rate is clearly maximized when the price is $p_{1}=19.74-\epsilon$, at a price slightly below the other provider's break-even price.

Example IV.2 In this example we consider a linear demand function $\sigma(p)=10-0.5 p$. The network parameters and thus the break-even price are the same as in the previous example, which we omit. Under this new and faster decreasing demand function, we plot the revenue rate in Figure 5(b). The revenue rate achieves its maximum at $p_{1}=15.76$ after which it demonstrates high-elasticity and starts to decrease with price. This results in the revenue maximizing price being less than $p_{2}^{B E}=19.74$. Therefore, facing such demand provider 1 would lower its price further below even though its competitor cannot match it without incurring a net loss, which demonstrates our result stated in Theorem IV.3(b).

Comparison with classical Bertrand duopoly. Theorem IV.3 essentially asserts that the equilibrium outcome of competition for secondary demand is a price war. Price wars are also typical outcomes in the classical Bertrand duopoly, hence it is worthwhile to put the two settings in perspective. In the Bertrand game, for a given price, both the revenue and the cost are linear functions of demand. In contrast, in the present setting neither revenue nor cost of secondary service are linear in secondary demand, primarily because both quantities rely 


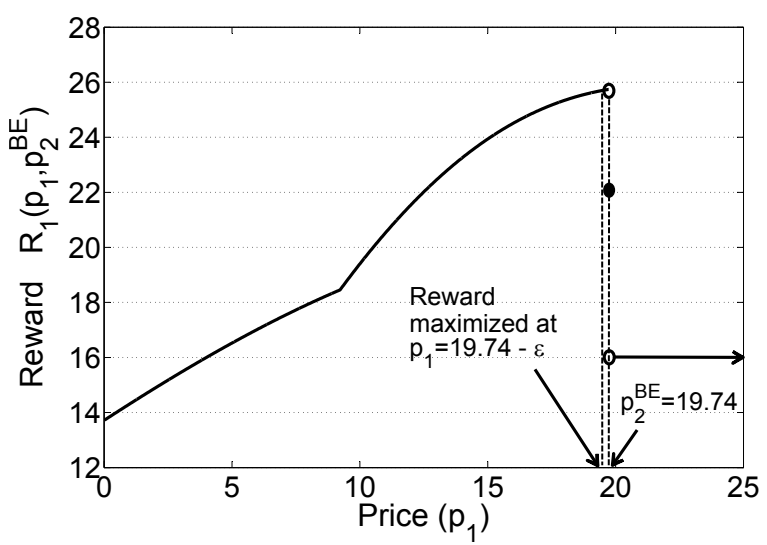

(a) Provider 1's reward maximized just below competitor's breakeven price when secondary demand is $\sigma\left(p_{1}\right)=10 e^{-0.2 p_{1}}$.

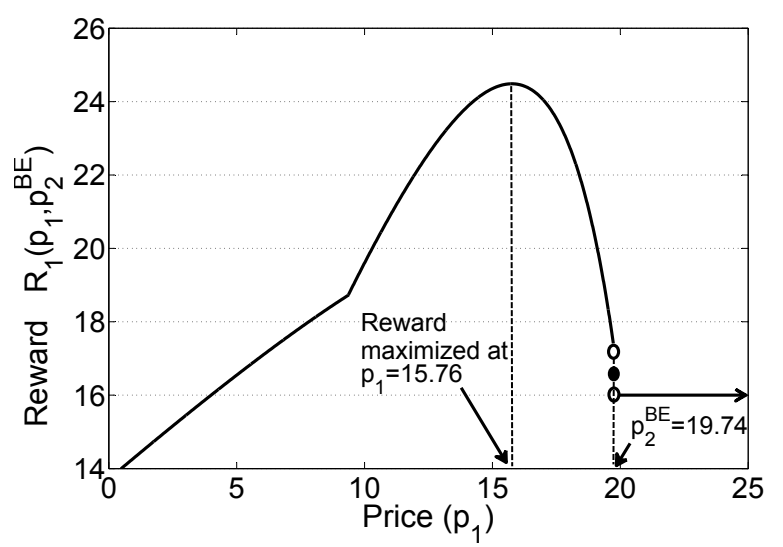

(b) Provider 1's reward maximized well below competitor's breakeven price when secondary demand is $\sigma\left(p_{1}\right)=10-0.5 p_{1}$.

Fig. 5: Different reward maximizing prices as provided in Examples IV.1 and IV.2.

heavily on blocking probabilities that are highly nonlinear in the demand. In addition, the Bertrand model precludes any capacity constraints and assumes that all demand can be satisfied, whereas the model of this paper is centered on a fundamental limitation in capacity. Yet, interestingly, the equilibrium of the present game resembles (and, depending on the secondary demand function, may be identical to) the outcome of a Bertrand game in which marginal cost is constant and equal to the break-even price.

This similarity is a consequence of two nontrivial properties established in the present paper: $(i)$ insensitivity of breakeven price against secondary demand, and (ii) Theorem IV.2, which indicates that having more secondary demand is always more favorable provided that secondary service is priced above break-even price. Both properties, however, rely on the assumption of optimal coordination of secondary access and does not necessarily extend to arbitrary access policies, as illustrated in the sequel.

Extension to multiple providers. Equilibrium strategy profiles given in Theorem IV.3 can be generalized to an arbitrary number of providers competing for the secondary demand, each with their own primary users, capacities and primary user rewards: Consider $N$ such providers and let $p_{i}^{B E}$ continue to represent the break-even price of provider $i$. Without any loss of generality, let us re-index the providers if necessary so that: $p_{1}^{B E} \leq p_{2}^{B E} \leq p_{3}^{B E} \leq \ldots \leq p_{N}^{B E}$.

Further we define $n=\max \left\{i: p_{i}^{B E}=p_{1}^{B E}\right\}$. Hence $n$ is the number of providers that share the lowest break-even price. We generalize the two cases presented in Theorem IV.3:

- If $n>1$ then any price profile $\left(p_{1}, p_{2}, \cdots, p_{N}\right)$ such that

$$
p_{i}=p_{1}^{B E} \quad \text { for } i=1,2, \cdots, n
$$

and

$$
p_{i}>p_{1}^{B E} \quad \text { for } i=n+1, n+2, \cdots, N .
$$

is a Nash equilibrium. In each such equilibrium providers $1,2, \cdots, n$ service the secondary demand at their break- even prices thereby generating no additional profit. The secondary demand is split among these providers according to an arbitrary probability vector $\left[\alpha_{1}, \alpha_{2}, \ldots, \alpha_{n-1}, \alpha_{n}\right]$ where $\sum_{i}^{n} \alpha_{i}=1, \alpha_{i}>0$, which has no bearing on equilibrium prices. The remaining $N-n$ providers are not able to capture any secondary demand.

- If $n=1$ then there is a single provider whose break-even price is lower than all the rest. In equilibrium this provider captures the entire secondary demand at a strictly profitable price, while the remaining $N-1$ providers cannot serve any secondary demand. In particular Nash equilibria have the form:

$$
p_{1} \in \mathcal{P},
$$

and

$$
p_{i} \geq p_{i}^{B E} \quad \text { for } i=2, \cdots, N,
$$

and at least one provider $j \neq 1$ chooses a price such that $p_{j}<p^{\max }$, where $p^{\max }$ is defined as in Eq. (21), so that there is no incentive for provider 1 to deviate from $\mathcal{P}$.

Quality of Service. QoS plays an important role wireless services. In this paper, QoS is implicitly captured through the implementation of a coordinated access policy. Under this policy, the QoS experienced by primary users will naturally be higher than that experienced by secondary users, since the provider reserves a certain part of its network capacity for the exclusive use of primary users. A possible refinement of the model is through the introduction of penalties. Specifically, whenever a provider is unable to accommodate a service request of an incoming user, it would compensate the blocked user by paying a fee (or giving a discount). If the penalty is imposed only when primary users are blocked (secondary access is opportunistic, and therefore has no associated penalties when blocked), then our results still hold through a similar analysis. 


\section{UnCOORdinated Access Policy}

In this section we consider equilibrium regimes that arise when competing providers grant uncoordinated access to secondary demand. We shall argue that such equilibria can be drastically different than those under an optimal coordinated access.

Under uncoordinated access, a provider does not differentiate between primary and secondary users in granting spectrum access requests. In turn, both types of users experience the same blocking probability. This probability depends on the aggregate demand and system capacity, and can be computed using standard techniques in teletraffic. Namely, when provider $i$ serves secondary demand $\sigma$, the two blocking probabilities are

$$
B_{i, 2}\left(\lambda_{i}, \sigma, A_{i}\right)=B_{i, 1}\left(\lambda_{i}, \sigma, A_{i}\right)=E\left(\lambda_{i}+\sigma, C_{i}\right),
$$

where $E\left(\lambda_{i}+\sigma, C\right)$ is the Erlang-B formula.

The revenue rate of provider $i$, when serving secondary demand $\sigma$ by charging $p_{i}$ per admitted request, is then given by

$$
\begin{aligned}
\hat{W}_{i}\left(p_{i}, \sigma\right)= & \left(1-E\left(\lambda_{i}+\sigma, C_{i}\right)\right) \sigma p_{i} \\
& +\left(1-E\left(\lambda_{i}+\sigma, C_{i}\right)\right) \lambda_{i} K_{i},
\end{aligned}
$$

where the first term corresponds to the reward rate collected from secondary users that gain admission to the network, while the second term corresponds to the reward rate collected from the serviced primary users. (Here and in the rest of this section we will consistently use the symbol ^ to indicate the quantities associated with uncoordinated access.) Once again, for analyses in which we consider a single provider, we will drop index $i$ from our notation for the sake of simplicity.

\section{A. Profitability}

We recognize $\hat{W}(p, 0)$ as the revenue rate of a provider when it does not serve any secondary demand. Similar to the profitability conditions for the optimal coordinated access case stated in Theorem IV.1, note that

$$
\hat{W}(p, \sigma(p)) \geq \hat{W}(p, 0)
$$

if and only if $p \geq \hat{p}^{B E}$, where $\hat{p}^{B E}$ satisfies:

$$
\hat{p}^{B E}=\frac{\left(E\left(\lambda+\sigma\left(\hat{p}^{B E}\right), C\right)-E(\lambda, C)\right) \lambda K}{\left(1-E\left(\lambda+\sigma\left(\hat{p}^{B E}\right), C\right)\right) \sigma\left(\hat{p}^{B E}\right)} .
$$

Hence the provider incurs loss and has no incentive to serve the secondary demand at a price below $\hat{p}^{B E}$. In turn $\hat{p}^{B E}$ is the break-even price of a provider under uncoordinated access.

It is instructive to compare the break-even prices under uncoordinated access and optimal coordinated access. Firstly, $\hat{p}^{B E} \geq p^{B E}$ because if the optimal admission policy does not yield positive profit from secondary demand then neither does any other policy. For typical parameters this inequality is strict. Consequently, providers need to charge a higher price to secondary users in order to avoid a net loss, which results in the tendency to bid higher prices under uncoordinated access. Secondly, in contrast to $p^{B E}$, the break-even price $\hat{p}^{B E}$ for uncoordinated access is given by an implicit equation that depends on the secondary demand $\sigma(p)$.

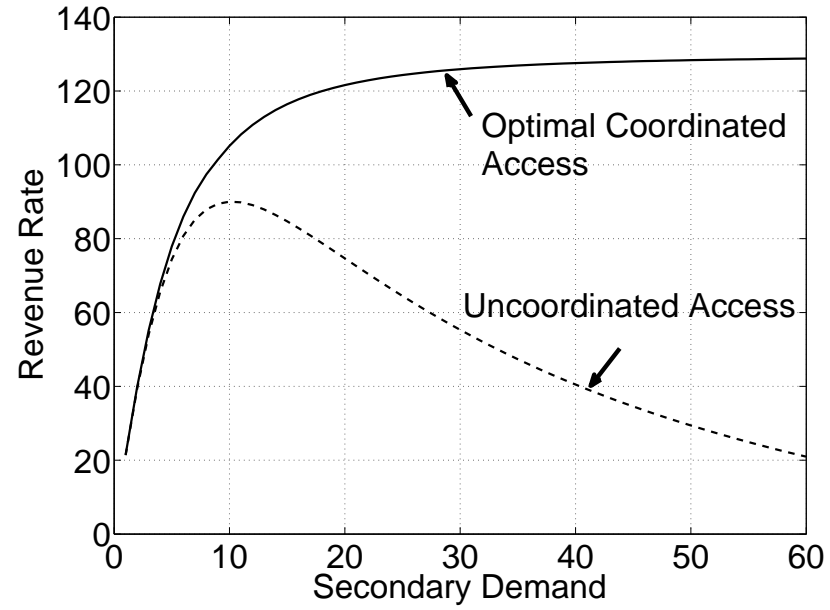

Fig. 6: Revenue rates under optimal coordinated and uncoordinated access versus secondary demand - network parameters: $p=30, \lambda=13, C=20$ and $K=50$.

\section{B. Market Sharing}

While we established in Lemma IV.2 that market sharing is not favorable under optimal coordinated access, these results do not necessarily extend to a case when uncoordinated access is implemented. As a matter of fact, under an uncoordinated access implementation, whether the revenue rate increases or decreases by sharing secondary demand depends on another critical parameter we shall establish later.

Before we get into our analysis, it is insightful to compare how the revenue rates $\hat{W}(p, \sigma)$ and $W^{*}(p, \sigma)$ behave under uncoordinated and optimal coordinated access strategies. Figure 6 illustrates the two revenue rates for a range of secondary demand $\sigma$,when all other parameters are fixed. When plotting both revenue rates, the secondary price $p$ is chosen above both break-even prices so that the optimal revenue rate $W^{*}(p, \sigma)$ is strictly increasing in $\sigma$. As a by-product of optimality, $W^{*}(p, \sigma) \geq \hat{W}(p, \sigma)$ under all circumstances.

However, $\hat{W}(p, \sigma)$ has an important qualitative difference relative to its optimal counterpart: It increases for a range of secondary demand $\sigma$ and decreases afterwards. This happens because for small $\sigma$, secondary users enhance revenue by using the leftover capacity from primary users, but as $\sigma$ increases secondary access occurs at an increasing expense of primary access and that leads to a decline in revenue if primary users are more valuable (that is, if $p<K$ ). This property opens the possibility that $\hat{W}(p, \alpha \sigma)>\hat{W}(p, \sigma)$, in which case a provider has incentive to share secondary demand at prices higher than break-even. Consequently, it has a profound impact on the outcome of a competitive setting.

To formalize this intuition let us define $p^{M S}$ as the solution to the following:

$$
p=\frac{(E(\lambda+\sigma(p), C)-E(\lambda+\alpha \sigma(p), C)) \lambda K}{(1-E(\lambda+\sigma(p), C)) \sigma(p)-(1-E(\lambda+\alpha \sigma(p))) \alpha \sigma(p)} .
$$


It can be verified directly from Eq. (22) that:

$$
\hat{W}(p, \alpha \sigma(p))\left\{\begin{array}{l}
>\hat{W}(p, \sigma(p)) \text { for } p<p^{M S} \\
\leq \hat{W}(p, \sigma(p)) \text { for } p \geq p^{M S} .
\end{array}\right.
$$

Thus, only up to the price value $p^{M S}$, any provider would benefit from a reduction in its secondary demand. The price $p^{M S}$ can be interpreted as a market sharing threshold for the provider: Any price above this threshold renders secondary demand too valuable to share and warrants a price war. Below this threshold, the provider has an incentive to remain at an equilibrium that reflects market sharing, provided the price satisfies the initial profitability condition in Eq. (23), which translates to being above the break-even price $\hat{p}^{B E}$.

\section{Profitable Sharing Interval}

In this section, we seek to determine the relationship between the maximum market sharing price $p^{M S}$ and the breakeven price $\hat{p}^{B E}$. In particular, if one can show that one price is always greater than the other, this can greatly simplify the results by ruling out or strictly establishing a preference to share the secondary market before making a negative profit. We present our results in the next theorem for the special case of fixed demand (we later present numerical evidence that similar results should hold for elastic demand):

Lemma V.1 For a fixed secondary demand such that $\sigma(p)=$ $\sigma$, the following price relationships always hold under an uncoordinated access policy:

$$
\hat{p}^{B E} \leq p^{M S}<K
$$

Proof:

a) First, we prove $\hat{p}^{B E} \leq p^{M S}$. Through Eqs. (24) and (25) we can rewrite this inequality in the following form:

$$
\begin{aligned}
& \frac{1-E(\lambda+\sigma(p), C)}{E(\lambda+\sigma(p), C)-E(\lambda, C)} . \\
& \frac{E(\lambda+\sigma(p), C)-E(\lambda+\alpha \sigma(p), C)}{1-E(\lambda+\sigma(p), C)-\alpha-\alpha E(\lambda+\alpha \sigma(p), C)} \geq 1 .
\end{aligned}
$$

Next, using the well-known recursive equation of the Erlang-B formula [24]:

$$
E(\lambda, C)=\frac{\lambda E(\lambda, C-1)}{C+\lambda E(\lambda, C-1)},
$$

we can expand Eq. (28) and after some algebra and regrouping of the terms, we can show that the inequality $\hat{p}^{B E} \leq p^{M S}$ is equivalent to demonstrating that:

$$
\begin{array}{r}
\alpha(\lambda+\sigma) E(\lambda+\sigma, C-1)+(1-\alpha) \lambda E(\lambda, C-1) \\
\geq(\lambda+\alpha \sigma) E(\lambda+\alpha \sigma, C-1) .
\end{array}
$$

Define $g_{C-1}(\lambda)=\lambda \cdot E(\lambda, C-1)$, which represents the traffic loss rate when the arrival process is Poisson with rate $\lambda$. For Eq. (29) to hold we need:

$$
\alpha g_{C-1}(\lambda+\sigma)+(1-\alpha) g_{C-1}(\lambda) \geq g_{C-1}(\lambda+\alpha \sigma) .
$$

Once can observe that Eq. (30) is by definition the convexity condition on the traffic loss as a function of the arrival rate, which is proven in [24]. Therefore, $\hat{p}^{B E} \leq p^{M S}$. b) We now show that the market sharing price is always less than the primary reward, i.e., $p^{M S}<K$. Recalling Eq. (25), this is equivalent to the following:

$$
\frac{\left(E(\lambda+\sigma, C)-E\left(\lambda+\alpha \sigma, C_{i}\right)\right) \lambda}{(1-E(\lambda+\sigma, C)) \sigma-(1-E(\lambda+\alpha \sigma)) \alpha \sigma}<1 .
$$

After some rearrangement of the terms and substituting $g_{C}(\lambda)$ for $\lambda \cdot E(\lambda, C)$, the inequality takes the form:

$$
g_{C}(\lambda+\sigma)-g_{C}(\lambda+\alpha \sigma)<\sigma(1-\alpha) .
$$

Upon careful observation, this inequality condition holds if one can show that:

$$
g_{C}^{\prime}(\lambda)=\frac{d g_{C}(\lambda)}{d \lambda}<1
$$

In the paper [24], it has been demonstrated that $g_{C}^{\prime}(\lambda) \leq 1$ for $C \geq 0$. The equality condition stems from the fact that the induction proof starts from $C=0$, for which $g_{C}(\lambda)=\lambda$ and hence $g_{C}^{\prime}(\lambda)=1$. If one would start the induction from $C=1$, using the following recursive formulation of $g_{C}(\lambda)$

$$
g_{C}(\lambda)=\frac{\lambda g_{C-1}(\lambda)}{C+\lambda g_{C-1}},
$$

provided in [24], one can show that $g_{1}(\lambda)=\lambda^{2} / 1+\lambda$. Taking the derivative with respect to $\lambda$,

$$
g_{1}^{\prime}(\lambda)=\frac{\lambda^{2}+2 \lambda}{\lambda^{2}+2 \lambda+1}<1 .
$$

Then following the same steps as in [24] one can show that $g_{C}^{\prime}(\lambda)<1$ for $C \geq 1$, which establishes Eq. (31).

Lemma V.1 establishes a fundamental relationship between the break-even and market sharing prices and the primary reward $K$, thus proving the existence of a profitable market sharing price interval. This interval plays a critical role in defining the market outcomes, as we shall demonstrate in the next section.

\section{Equilibrium}

Competitive equilibria under uncoordinated access can now be determined depending on the critical price values $\hat{p}_{i}^{B E}$ and $p_{i}^{M S}$ of all providers $i$. Figure 7 illustrates a particular placement of these parameters for two providers. In the illustrated setting, the market sharing intervals $\left[\hat{p}_{1}^{B E}, p_{1}^{M S}\right]$ and $\left[\hat{p}_{2}^{B E}, p_{2}^{M S}\right]$ have a non-empty intersection; therefore there exist common price values that are above break-even values and acceptable for market sharing for both providers. In turn, there is a continuum of equilibria strictly above the break-even prices.

We conclude this section with a numerical example concerning a symmetric setting.

Example V.1 We consider two network providers with identical parameters: Primary arrival rate $\lambda_{i}=13$, capacity $C_{i}=20$, and revenue collected per serviced primary user $K_{i}=50$. We continue to assume inelastic secondary demand whose value is chosen to be $\sigma=20$. We assume that secondary demand splits equally in the case of equal prices, that is, $\alpha_{1}=\alpha_{2}=0.5$. 


\begin{tabular}{|c||c|c|}
\hline Access Policy & Equilibrium price & Equilibrium profit \\
\hline Coordinated & $p_{1}=p_{2}=0.91$ & $P_{1}=P_{2}=0$ \\
Uncoordinated & $23.46 \leq \hat{p}_{1}=\hat{p}_{2} \leq 34.11$ & $0 \leq \hat{P}_{1}=\hat{P}_{2} \leq 121.54$ \\
\hline
\end{tabular}

TABLE I: Equilibrium prices and resulting profits for the setting considered in Example V.1.

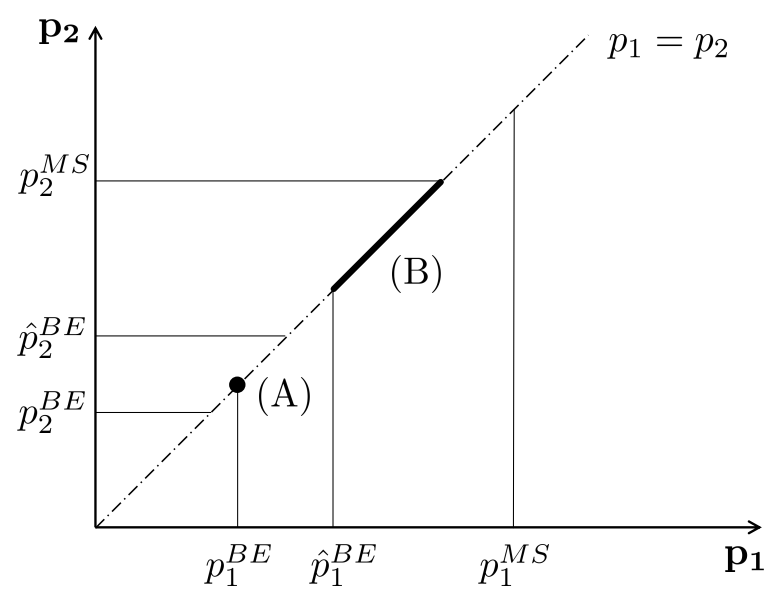

Fig. 7: Point (A) represents the highest price Nash equilibrium under optimal coordinated access, and the continuum of points in (B) is the set of Nash equilibria under uncoordinated access.

The break-even price for coordinated access is computed as 0.91 ; hence by Theorem IV.3 the unique price equilibrium under coordinated access is $p_{1}=p_{2}=0.91$ and no provider profits from secondary demand.

The break-even price for uncoordinated access is $\hat{p}_{i}^{B E}=$ 23.46 and the market sharing threshold is $p_{i}^{M S}=34.11$. Hence, any price profile $(p, p)$ where $p$ lies in the interval $[23.46,34.11]$ constitutes a competitive equilibrium. For example, if provider $-i$ adopts the secondary price $p_{-i}=30$ then

$$
R_{i}\left(p_{1}, p_{2}\right)= \begin{cases}\hat{W}_{i}\left(p_{i}, \sigma\right)=74.66 & \text { if } p_{i}=29.99 \\ \hat{W}_{i}\left(p_{i}, 0.5 \sigma\right)=90.01 & \text { if } p_{i}=30 \\ \hat{W}_{i}\left(p_{i}, 0\right)=0 & \text { if } p_{i}>30\end{cases}
$$

In particular $p_{i}=30$ is the best response of provider $i$; and so the price profile $(30,30)$ is a Nash equilibrium. A comparison of possible equilibria and associated profits under both access strategies is given in Table I. It is worth noting that in the coordinated access policy, the price war drives the profits of both provider to zero by lowering the prices to the break-even price, which is the same for each provider. On the other hand, uncoordinated access gives a range of prices yielding positive profits in the sharing interval. Note that profit from primary users is not included in either case.

Interestingly, an uncoordinated access policy, which is suboptimal to implement for a provider in isolation, results in competitive equilibria in which all providers are strictly better off than resorting to their optimal individual policies.
Example V.2 This time, we consider an elastic demand to demonstrate that our results extend beyond inelastic secondary demand. Once again there are two network providers with identical parameters: Primary arrival rate $\lambda_{i}=30$, capacity $C_{i}=50$, and revenue collected per serviced primary user $K_{i}=50$. We assume a secondary demand that is exponentially decreasing with the price $\sigma(p)=80 e^{-0.02 p}$. We assume that secondary demand splits equally in the case of equal prices, that is, $\alpha_{1}=\alpha_{2}=0.5$.

The break-even price for coordinated access is computed as 0.01 ; hence by Theorem IV.3 the unique price equilibrium under coordinated access is $p_{1}=p_{2}=0.01$ and no provider profits from secondary demand.

The break-even price for uncoordinated access is $\hat{p}_{i}^{B E}=$ 20.06 and the market sharing threshold is $p_{i}^{M S}=33.39$. Hence, any price profile $(p, p)$ where $p$ lies in the interval $[20.06,33.39]$ constitutes a competitive equilibrium. The same arguments discussed within Example V.1 also apply here.

\section{CONCLUSION}

In this paper we provided results on the competitive nature of a secondary spectrum market with multiple firms by investigating the equilibrium outcomes. Our focus was on two proposed regimes for secondary spectrum access, namely coordinated access and uncoordinated access under private commons. This kind of market analysis can help provide important guidance to a firm's strategic decision process by explicitly determining the parameters on which market success depends. Given the current state of the wireless industry, this research may encourage the adoption of dynamic sharing technologies. To achieve this goal, we formulated the problem as a non-cooperative game, in which network providers with finite spectral capacities choose price and access control strategies to follow with respect to secondary users.

We started our analysis with a threshold type optimal coordinated access policy. We demonstrated that in a secondary market each provider has a unique break-even price, which serves as the minimum price at which profitability of secondary provisions is guaranteed regardless of the secondary user demand. We provided an explicit formula to calculate the break-even price, which also establishes its relationship with the network parameters (i.e., the primary load, primary reward, and system capacity). The break-even price of each provider is independent of the system parameters of other providers. The break-even price is, in general, significantly lower than the primary reward, indicating that secondary access can be offered for relatively low prices. For example, the break-even price is less than $1 \%$ of the primary reward if the network load is below $68 \%$ and the number of channels $C$ exceeds 32 . Even at the critical load where the primary load is equal to the 
system capacity (i.e., $\lambda=C$ ), the break-even price remains below $20 \%$ of the primary reward for $C \geq 16$.

Next, we provided a detailed study of the strictly increasing property of the revenue rate under optimal coordinated access through threshold type control and unimodality of the revenue function for each threshold.. This lead to the establishment of the best response for a network provider which is to always underbid the competition as long as it finds it profitable to do so. In the presence of multiple firms, this pricing strategy results in a price war where a single provider (that with the lowest break-even price) captures the entire secondary spectrum market, effectively forming a monopoly. We then listed the possible market outcomes using the notion of Nash equilibrium in a non-cooperative game where two or more network providers implement an optimal coordinated access policy. While the demand function does not play a role in determining the identity of the winning provider, we showed that is does affects the revenue-maximizing price for that provider and the placement of the Nash equilibrium.

Finally, we showed that the market dynamics fundamentally differ when providers implement uncoordinated access. We highlighted that the break-even price is no longer insensitive to secondary demand and market sharing becomes a possible best response, determined by another price threshold. Furthermore, we demonstrated the existence of a profitable sharing price interval for fixed secondary demand (we verified numerically that the profitable sharing interval exists for other forms of secondary demand). It is worth noting that even though a provider might find it desirable to share the market, it would still go into a price war for price values higher than its market sharing price $p^{M S}$, thus preventing convergence to an arbitrarily high price for secondary access. The possible market outcomes under an uncoordinated access policy become complex for general forms of demand functions, but deserve further study, since they may result in a larger number of providers participating the a secondary spectrum market as well as the realization of higher revenues rates than possible under an optimal coordinated access policy.

\section{APPENDIX}

Proof of Theorem IV.3: We will prove the the two parts of Theorem IV.3 separately, first when $p_{1}^{B E}<p_{2}^{B E}$ and second when $p_{1}^{B E}=p_{2}^{B E}$. Under each case, we will demonstrate that the price pairs described in the theorem give the Nash equilibria by proving that neither provider $i=1,2$ can increase its reward $R_{i}\left(p_{1}, p_{2}\right)$ by employing any other strategy profile.

Part $1-p_{1}^{B E}<p_{2}^{B E} \quad$ In a given Nash equilibrium the pricing strategy each provider chooses is given by:

$$
p_{1} \in \mathcal{P}
$$

and

$$
p_{2} \in\left(p_{1}, p^{\max }\right) .
$$

Under these strategies provider 1's reward is

$$
R_{1}\left(p_{1}, p_{2}\right)=\bar{W}_{1}^{*}>W_{1}^{*}\left(p_{1}, 0\right),
$$

where $\bar{W}_{1}^{*}$ is given by Eq. (19) and $W_{1}^{*}\left(p_{1}, 0\right)$ represents the base revenue rate provider 1 collects from the primary users in the absence of secondary users. Thus provider 1 collects a positive revenue from capturing the entire secondary market above its break-even price. On the other hand, provider 2 is unable to attract any secondary demand and faces the reward:

$$
R_{2}\left(p_{1}, p_{2}\right)=W_{2}^{*}\left(p_{2}, 0\right) .
$$

We first analyze the possible increases in reward when provider 2 chooses other price strategies.

Suppose provider 2 chooses any price $p_{2}^{\prime}<p_{1}$. Then provider 2 captures the secondary demand but since $p_{2}^{\prime}<$ $p_{1}<p_{2}^{B E}$ this is a non-profitable price. Hence provider 2 choose to implement a lock-out policy which is reflected in the reward:

$$
R_{2}\left(p_{1}, p_{2}^{\prime}\right)=W_{2}^{*}\left(p_{2}^{\prime}, \sigma\left(p_{2}^{\prime}\right)\right)=W_{2}^{*}\left(p_{2}, 0\right),
$$

by Theorem IV.1. Therefore $R_{2}\left(p_{1}, p_{2}^{\prime}\right)=R_{2}\left(p_{1}, p_{2}\right)$.

Now suppose provider 2 chooses any price $p_{2}^{\prime} \geq p^{\max }$, which we have previously defined in Eq. (21). This action does not change the reward of provider 2 as it remains in a position where it capture no secondary demand. Hence, $R_{1}\left(p_{1}, p_{2}^{\prime}\right)=$ $R_{1}\left(p_{1}, p_{2}\right)$.

Having proven provider 2 has no incentive to deviate, we shift our focus to provider 1.

If provider 1 chooses a price $p_{1}^{\prime}>p_{2}$, this results in the loss of the secondary demand and its reward becomes $R_{1}\left(p_{1}^{\prime}, p_{2}\right)=$ $W_{1}^{*}\left(p_{1}^{\prime}, 0\right)=W_{1}^{*}\left(p_{1}, 0\right)<R_{1}\left(p_{1}, p_{2}\right)$.

If provider 1 chooses a price $p_{1}^{\prime}=p_{2}$, it shares the secondary demand with provider 2 and its reward becomes $R_{1}\left(p_{1}^{\prime}, p_{2}\right)=W_{1}^{*}\left(p_{2}, \alpha_{1} \sigma\left(p_{2}\right)\right)$. By Theorem IV.2 there exists an $\epsilon>0$ such that:

$$
W_{1}^{*}\left(p_{2}, \alpha_{1} \sigma\left(p_{2}\right)\right)<W_{1}^{*}\left(p_{2}-\epsilon, \sigma\left(p_{2}-\epsilon\right)\right),
$$

hence $R_{1}\left(p_{1}^{\prime}, p_{2}\right)<R_{1}\left(p_{1}, p_{2}\right)$.

If provider 1 chooses a price $p_{2}^{B E} \leq p_{1}^{\prime}<p_{2}$, this implies through Eq. (34) that $p_{1}^{\prime}<p^{\max }$. By the definition of $p^{\max }$ in Eq. (21), for any price $p_{1}^{\prime}<p^{\max }$ we have:

$$
W_{1}^{*}\left(p_{1}^{\prime}, \sigma\left(p_{1}^{\prime}\right)\right) \leq \bar{W}_{1}^{*} .
$$

Hence $R_{1}\left(p_{1}^{\prime}, p_{2}\right)=W_{1}^{*}\left(p_{1}^{\prime}, \sigma\left(p_{1}^{\prime}\right)\right)<R_{1}\left(p_{1}, p_{2}\right)$.

If provider 1 chooses a price $p_{1}^{\prime}<p_{1}^{B E}$, it serves secondary demand at a non-profitable price and hence faces the reward $R_{1}\left(p_{1}^{\prime}, p_{2}\right)=W_{1}^{*}\left(p_{1}^{\prime}, \sigma\left(p_{1}^{\prime}\right)\right)=W_{1}^{*}\left(p_{1}, 0\right)<R_{1}\left(p_{1}, p_{2}\right)$.

Finally, if provider 1 chooses a price $p_{1}^{\prime} \in\left[p_{1}^{B E}, p_{2}^{B E}-\epsilon\right]$ but $p_{1} \notin \mathcal{P}$, from the way $\mathcal{P}$ is defined, the new reward is $R_{1}\left(p_{1}^{\prime}, p_{2}\right)=W_{1}^{*}\left(p_{1}^{\prime}, \sigma\left(p_{1}^{\prime}\right)\right)<\bar{W}_{1}^{*}$. Therefore $R_{1}\left(p_{1}^{\prime}, p_{2}\right)<R_{1}\left(p_{1}, p_{2}\right)$

Part $2-p_{1}^{B E}=p_{2}^{B E} \quad$ Since both providers are identical, we will only consider provider 1 . Also, for the sake of notational simplicity we will drop the index on the break-even price and denote it by $p^{B E}$. Provider 1 , when at the Nash equilibrium, chooses the price strategy $p_{1}=p^{B E}$ and faces the reward $R_{1}\left(p_{1}, p_{2}\right)=W_{1}^{*}\left(p_{1}, 0\right)$.

We fix provider 2's strategy to $p_{2}=p^{B E}$ and demonstrate that provider 1's reward does not improve by choosing any 
other action pair.

If provider 1 chooses a pricing strategy $p_{1}^{\prime}>p^{B E}$, it faces a reward $R_{1}\left(p_{1}^{\prime}, p_{2}\right)=W_{1}^{*}\left(p_{1}, 0\right)=R_{1}\left(p_{1}, p_{2}\right)$.

If provider 1 chooses any pricing strategy $p_{1}^{\prime}<p^{B E}$, by definition of $p^{B E}$ it faces a reward $R_{1}\left(p_{1}^{\prime}, p_{2}\right)=$ $W_{1}^{*}\left(p_{1}, \sigma\left(p_{1}\right)\right)=W_{1}^{*}\left(p_{1}, 0\right)=R_{1}\left(p_{1}, p_{2}\right)$.

Because of provider symmetry, the same proof follows for player 2.

Therefore the only Nash equilibrium is given by the price pair $p_{1}^{B E}=p_{2}^{B E}$, from which uniqueness also follows since the break-even price of each provider is unique.

Having shown that under both cases Nash equilibria exist and can not be different from what is stated in Theorem IV.3, we conclude our proof.

\section{REFERENCES}

[1] The Federal Communications Commission, Promoting Efficient Use of Spectrum Through Elimination of Barriers to the Development of Secondary Markets. http://hraunfoss.fcc.gov/edocs_public/attachmatch/ FCC-04-167A1.pdf

[2] Shared Spectrum Company, General Survey of Radio Frequency Bands (30 $\mathrm{MHz}$ to $3 \mathrm{GHz}$ ): Vienna, Virginia, September 1-5, 2009. http://www.sharedspectrum.com/wp-content/uploads/2010 0923-General-Band-Survey-30MHz-to-3GHz.pdf

[3] I.F. Akyildiz, W.Y. Lee, M.C. Vuran, and S. Mohanty. Next generation/dynamic spectrum access/cognitive radio wireless networks: a survey. Computer Networks, 50(13):2127-2159, 2006.

[4] M. Alanyali, A. Al Daoud, D. Starobinski. Profitability of dynamic spectrum provision for secondary use. In DySPAN, May 2011.

[5] M. Alanyali, A. Al Daoud, D. Starobinski. Economic Viability of Private Commons: Framework and Guidelines for Profitability. Telecommunications Policy, 37(2):231-240, 2013.

[6] J. Bae, E. Beigman, R. Berry, M.L. Honig, H. Shen, R. Vohra, and H. Zhou. Spectrum markets for wireless services. In DySPAN, pages 1-10, Chicago, October 2008.

[7] D. Bertsekas. (1976) Dynamic Programming and Stochastic Control. Academic Press, Inc.

[8] M.M. Buddhikot. Understanding dynamic spectrum access: Models, taxonomy and challenges. In DySPAN, pp.649-663, 2007.

[9] M. Bykowsky. A secondary market for the trading of spectrum: promoting market liquidity. Telecommunications Policy, 27(7):533 -541, 2003.

[10] M. Bykowsky, M. Olson, and W. Sharkey. Efficiency gains from using a market approach to spectrum management. Information Economics and Policy, 22(1):73-90, 2010.

[11] J.M. Chapin, and W.H. Lehr. The path to market success for dynamic spectrum access technology. IEEE Communications Magazine, 45(5):96103, May 2007.

[12] L. Duan, J. Huang, and B. Shou. Competition with dynamic spectrum leasing. In CoRR, abs/1003.5517, 2010.

[13] A. Harel. Sharp Bounds and Simple Approximations for the Erlang Delay and Loss Formulas. Management Science, 34(8):959-972, August 1988.

[14] O. Ileri, D. Samardzija, and N.B. Mandayam. Demand responsive pricing and competitive spectrum allocation via a spectrum server. In DySPAN, pp. 194-202, 8-11 2005.

[15] K. Jagannathan, I. Menache, E. Modiano, G. Zussman. Noncooperative Spectrum Access - The Dedicated vs. Free Spectrum Choice. In MobiHoc, May 2011.

[16] A.A Jagers, E. A. Van Doorn. On the continued Erlang loss function. Operations Research Letters, 5(1):43-46, June 1986.
[17] A. J. E. M. Janssen, J. S. H. Van Leeuwaarden, B. Zwart. Gaussian Expansions and Bounds for the Poisson Distribution Applied to the Erlang B Formula Advances in Applied Probability, 40(1):122-143, March 2008.

[18] W.J. Kaczor, M.T. Nowak. (2000) Problems in Mathematical Analysis II. American Mathematical Society, Providence, RI.

[19] G.S. Kasbekar, S. Sarkar. Spectrum pricing games with arbitrary bandwidth availability probabilities. In ISIT, July 2011.

[20] E. Kavurmacioglu, M. Alanyali, D. Starobinski. Competition in Secondary Spectrum Markets: Price War or Market Sharing? In DySPAN, October 2012

[21] H. Kim, J. Choi, and K.G. Shin. Wi-Fi 2.0: Price and Quality Competitions of Duopoly Cognitive Radio Wireless Service Providers with Time-varying Spectrum Availability. In INFOCOM 2011.

[22] P. Key. Optimal control and trunk reservation in loss networks. Probability in the Engineering and Informational Sciences, 4:203-242, 1990.

[23] O. Korcak, T. Alpcan, and G. Iosifidis. Collusion of Operators in Wireless Spectrum Markets. In WiOpt 2012.

[24] K. R. Krishnan. The convexity of loss rate in an Erlang loss system and sojourn in an Erlang delay system with respect to arrival and service rates. IEEE Transactions on Communications, 38(9):1314-1316, September 1990.

[25] P. Maille, B. Tuffin. Price war in heterogeneous wireless networks. Computer Networks, 54(13):2281-2292, September 2010.

[26] P. Maille, B. Tuffin, J.M. Vigne. Technological investment games among wireless telecommunications service providers. International Journal of Network Management, 21(1) 2008.

[27] J.W. Mayo, and S. Wallsten. Enabling efficient wireless communications: The role of secondary spectrum markets. Information Economics and Policy, 22(1):61-72, 2010.

[28] M. Michalopoulou, J. Riihijarvi, and P. Mahonen. Towards Characterizing Primary Usage in Cellular Networks: A Traffic-based Study. In DYSPAN, Aachen Germany, May 2011.

[29] B. Miller. A queueing reward system with several customer classes. Management Science, 16:234-245, 1971.

[30] H. Mutlu, M. Alanyali, and D. Starobinski. Spot pricing of secondary spectrum usage in wireless cellular networks. IEEE/ACM Transactions on Networking, 17(6):1794-1804, December 2009.

[31] H. Mutlu, M. Alanyali, and D. Starobinski. On-line Pricing of Secondary Spectrum Access with Unknown Demand Function and Call Length Distribution. In INFOCOM 2010, March 2010.

[32] D. Niyato, E. Hossain. Competitive Pricing for Spectrum Sharing in Cognitive Radio Networks: Dynamic Game, Inefficiency of Nash Equilibrium, and Collusion. Selected Areas in Communications, IEEE Journal on , 26(1):192-202, January 2008

[33] M. J. Osbourne. (2004) pp. 64-67 An Introduction to Game Theory. Oxford University Press, New York, NY, USA.

[34] R. Ramjee, D. Towsley, and R. Nagarajan. On optimal call admission control in cellular networks. Wireless Networks, 3:29-41, 1997.

[35] Y. Ren, Q. Zhang, P. Chen. Cooperative and Non-cooperative WSPs in Dynamic Spectrum Leasing. In Communications and Mobile Computing pp. 274-277, 2011.

[36] S. Sengupta, and M. Chatterjee. An economic framework for dynamic spectrum access and service pricing. Networking, IEEE/ACM Transactions on, 17(4): 1200-1213, August 2009.

[37] K. T. Talluri, G. V. Ryzin (2004) pp. 321-323 The Theory and Practice of Revenue Management. Springer Science+Business Media, New York, NY, USA.

[38] G. J. Tellis, and G. J. Gaeth. Best Value, Price-Seeking, and Price Aversion: The Impact of Information and Learning on Consumer Choices. Journal of Marketing, 54(2):34-45, April 1990.

[39] Y. Xing, R. Chandramouli, and C. Cordeiro. Price dynamics in competitive agile spectrum access markets. Selected Areas in Communications, IEEE Journal on, 25(3):613-621, April 2007. 